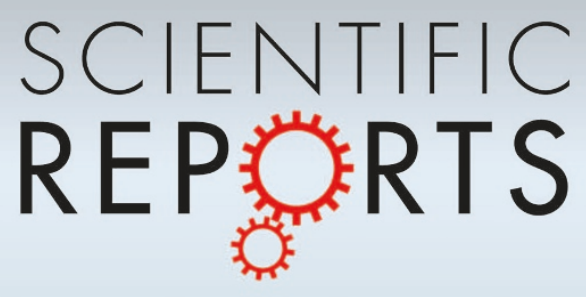

\title{
OPEN Average structure and local configuration of excess oxygen in $\mathrm{UO}_{2+x}$
}

SUBJECT AREAS:

ATOMISTIC MODELS

STRUCTURE OF SOLIDS AND

LIQUIDS

CERAMICS

MATERIALS FOR ENERGY AND

CATALYSIS

Received

27 November 2013

Accepted

31 January 2014

Published

19 March 2014

Correspondence and requests for materials should be addressed to J.W.W. Jianwei@lsu. edu)

\author{
Jianwei Wang ${ }^{1,3}$, Rodney C. Ewing ${ }^{2,3}$ \& Udo Becker ${ }^{3}$
}

'Department of Geology and Geophysics, Center for Computation and Technology, Louisiana State University, Baton Rouge, Louisiana 70803-0001, USA, '2Department of Geological and Environmental Sciences, Stanford University Stanford, California 94305-21 15, USA, ${ }^{3}$ Department of Earth and Environmental Sciences, University of Michigan, Ann Arbor, Michigan 48109-1005, USA.

Determination of the local configuration of interacting defects in a crystalline, periodic solid is problematic because defects typically do not have a long-range periodicity. Uranium dioxide, the primary fuel for fission reactors, exists in hyperstoichiometric form, $\mathrm{UO}_{2+\mathrm{x}}$. Those excess oxygen atoms occur as interstitial defects, and these defects are not random but rather partially ordered. The widely-accepted model to date, the Willis cluster based on neutron diffraction, cannot be reconciled with the first-principles molecular dynamics simulations present here. We demonstrate that the Willis cluster is a fair representation of the numerical ratio of different interstitial $\mathrm{O}$ atoms; however, the model does not represent the actual local configuration. The simulations show that the average structure of $\mathrm{UO}_{2+x}$ involves a combination of defect structures including split di-interstitial, di-interstitial, mono-interstitial, and the Willis cluster, and the latter is a transition state that provides for the fast diffusion of the defect cluster. The results provide new insights in differentiating the average structure from the local configuration of defects in a solid and the transport properties of $\mathrm{UO}_{2+\mathrm{x}}$.

ranium dioxide is the principal fuel of nuclear reactors. One of the unique properties of $\mathrm{UO}_{2}$ is its ability to accommodate a variable stoichiometry, depending on temperature and oxygen pressure ${ }^{1,2}$. The excess oxygen atoms in hyperstoichiometric uranium dioxide $\left(\mathrm{UO}_{2+\mathrm{x}}\right)$ occur as interstitial defects ${ }^{3-11}$. Positions and dynamics of these excess oxygen atoms control many important properties, such as thermal conductivity ${ }^{12-14}$, fission-product accommodation and transport ${ }^{15}$, micro-structure evolution ${ }^{16,17}$, and corrosion behavior ${ }^{18,19}$. These properties are closely related to the performance of the fuel in a reactor and its behavior in a geologic disposal. For $\mathrm{UO}_{2+\mathrm{x}}$ at low $\mathrm{x}$ values, the interstitial $\mathrm{O}$ atoms occur as isolated point defects. As $\mathrm{x}$ increases, individual defects interact with each other increasingly and form clusters ${ }^{3-5,20,21}$. Various experimental and theoretical studies have shown that these clusters are not random but rather structured with well-defined configurations ${ }^{3-6,11,22,23}$. However, the defect structures are difficult to quantify experimentally using diffraction techniques because the clusters are local structures and lack long-range periodicity. Based on early neutrondiffraction studies, a defect cluster model, the so-called $2: 2: 2$ Willis type, was proposed for $\mathrm{UO}_{2.11-2.13}$ over fifty years $\mathrm{ago}^{3}$. Since then, this has remained the dominant conceptual model in the literature. Although slightly modified later by the original author and the collaborators $s^{4,5}$, the proposed oxygen configuration remains the same $^{4,5}$. The acceptance of the model is largely based on the neutron diffraction data. However, recent firstprinciples calculations and empirical potential molecular dynamics show that the Willis cluster is not stable $\mathrm{e}^{11,22-25}$. Upon optimization, it spontaneously relaxes to a split di-interstitial or tri-oxygen cluster sharing a vacancy (V3O") ${ }^{11,22-25}$. The essential question is whether the Willis defect is an appropriate model for $\mathrm{UO}_{2+\mathrm{x}}$ or whether it is a limitation of static first-principles calculations being performed at the athermal limit that cannot account for finite temperature effects on the defect structure. In order to overcome the limitations related to zero temperature and the accuracy of empirical potentials in the previous theoretical calculations ${ }^{11,22-25}$, first-principles moleculardynamics simulations at high temperatures are employed here. These results provide a self-consistent explanation of the Willis cluster model that is based on neutron diffraction data and the atomistic-scale structure that is derived from recent theoretical calculations. The simulations also improve the understanding of different defect types and reveal the role of the Willis defect model in the transport of the oxygen defect clusters in $\mathrm{UO}_{2+\mathrm{x}}$.

\section{Results and discussion}

Uranium dioxide has the isometric fluorite structure $(F m 3 m)$. The 4 a site is occupied by uranium, the $8 \mathrm{c}$ site by oxygen, and $4 \mathrm{~b}$ site is empty in $\mathrm{UO}_{2}$. The Willis $2: 2: 2$ defect cluster consists of two vacancies $\left(\mathrm{V}_{\mathrm{o}}\right)$ at the $\mathrm{O} 8 \mathrm{c}$ site, two $\mathrm{O}$ interstitials displaced in $<110>$ directions $\left(\mathrm{O}^{\prime}\right)$ from the $4 \mathrm{~b}$ site, and two other $\mathrm{O}$ interstitials displaced in $<111>\left(\mathrm{O}^{\prime \prime}\right)$ from the $4 \mathrm{~b}$ site (Figure $\left.1 \mathrm{a}\right)^{3-5}$. Starting with this configuration without constraints, the cluster was 
optimized to a split di-interstitial defect (V3O"). Depending on how the Willis defect is perturbed by a small change in geometry, one of the $<111>$ oxygen interstitials (O") moves back to one vacant lattice $8 \mathrm{c}$ site while the other O" and two O' move away from their initial positions towards to the other vacant lattice $8 \mathrm{c}$ site. This split diinterstitial defect (V3O") consists of three interstitial O atoms sharing an oxygen $8 \mathrm{c}$ site. This result confirms the instability of the Willis defect cluster repeatedly reported in recent first-principles studies using similar computational methods ${ }^{22-25}$. Geometry optimizations starting from the Willis defect configuration (Figure 1a) and with a constraint to allow the two $\langle 110\rangle \mathrm{O}$ atoms to move only in the (001) plane result in a only slightly modified Willis cluster configuration. However, when the constraint is removed, the structure relaxes to a split di-interstitial defect. This result suggests that, although the Willis cluster is unstable, it could provide a transition path for cluster migration from one location to another, very different from previously studied paths for oxygen di-interstitial diffusion $^{11,23,25}$. In order to check the energy barrier of the transition path, the Climbing Image-Nudged Elastic Band (CI-NEB) method ${ }^{26}$ was applied to estimate the barrier for the Willis cluster configuration
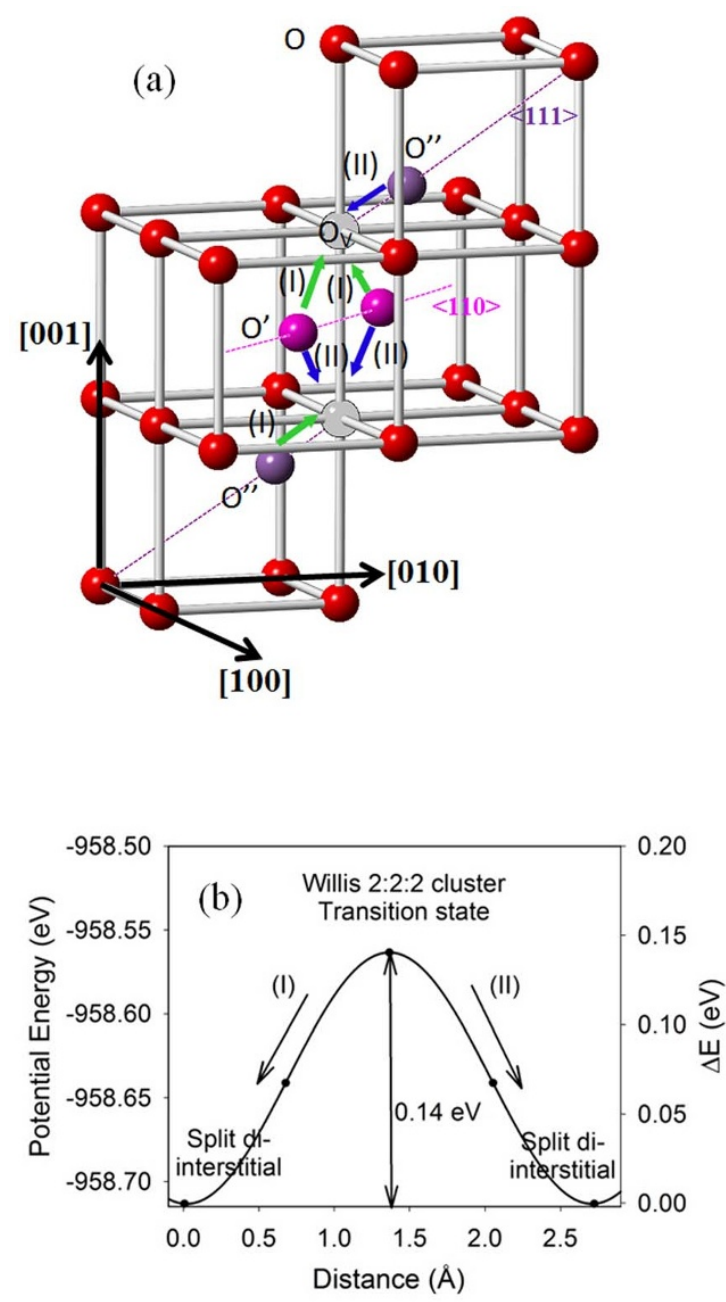

Figure 1 Schematic structure of Willis 2:2:2 defect cluster (a), which relaxes to either one of the split di-interstitial defects, depending on the path I or II respectively. The arrows indicate the direction of the movements of the atoms. (b) Potential energy surface of the migration of a split di-interstitial defect cluster. Migration energy barrier: $0.14 \mathrm{eV}$. Arrows in (b) show relaxation directions. Spheres are O atoms. Uranium atoms are not shown. Red stands for lattice $8 \mathrm{c} \mathrm{O}$ atoms, purple and pink for oxygen interstitials displaced along $<111><110>$, respectively, and grey circles for the oxygen vacancy at the $8 \mathrm{c}$ site. as a transition state. The calculated migration energy is $\sim 0.14 \mathrm{eV}$ (Figure $1 \mathrm{~b}$ ), which is low, about 5 times $\mathrm{k}_{\mathrm{B}} \mathrm{T}$ at ambient temperature. Similar migration energies were estimated using empirical potential models and temperature-accelerated molecular-dynamics simulations but assuming different transition paths ${ }^{11,25}$. A recent DFT calculation estimated a higher migration barrier of $0.47 \mathrm{eV}$ for a split diinterstitial assuming a different path ${ }^{23}$. Since static diffusion calculations often have to presume a transition path, one may question whether the paths and calculated values at the athermal limit represent the properties of the diffusion at the finite temperatures at which experiments with $\mathrm{UO}_{2+\mathrm{x}}$ are often performed.

In order to reconcile the structure of the Willis defect and to directly reveal the migration of a split di-interstitial defect cluster, first-principles molecular-dynamics (MD) simulations were carried out at $300 \mathrm{~K}, 500 \mathrm{~K}, 800 \mathrm{~K}, 1200 \mathrm{~K}, 1600 \mathrm{~K}$, and $2000 \mathrm{~K}$. It was expected that thermal energy at higher temperatures activates the cluster migration and increases the effectiveness of probing the phase space of the cluster migration, giving its low migration energy barrier. The simulations started with one split di-interstitial in the computational supercell $\left(\mathrm{UO}_{2.06}\right)$. At low temperatures $(300 \mathrm{~K}-800 \mathrm{~K})$, the split di-interstitials remain at their initial location with a configuration of the two interstitials displaced from the octahedral $4 \mathrm{~b}$ sites, pushing a lattice $8 \mathrm{c}$ oxygen atom towards the other octahedral $4 \mathrm{~b}$ site, resulting in three interstitials sharing an oxygen lattice $8 \mathrm{c}$ site. Figure $2 \mathrm{a}$ shows the atomic density probability contour maps at $300 \mathrm{~K}$ projected on (001) plane. Similar results were observed at $500 \mathrm{~K}$ and $800 \mathrm{~K}$. At $1200 \mathrm{~K}$, one of the interstitial $\mathrm{O}$ atoms is moved back to its lattice site while another oxygen interstitial appears, resulting in a new split di-interstitial defect cluster at a different location (Figure 2b). At $1600 \mathrm{~K}$, the cluster has become two separate mono-interstitials (Figure 2c). After the dissociation, the two monointerstitial defects remain relatively immobile for the rest of the molecular dynamics simulation. As the temperature increases to $2000 \mathrm{~K}$, the cluster becomes highly mobile and goes through multiple transformations at different locations (Figure 2d).

A careful analysis of the trajectory of $\mathrm{UO}_{2.06}$ at $2000 \mathrm{~K}$ shows that, as the split di-interstitial cluster migrates, it is transformed from one to another of the same kind. During the transition, the defect cluster passes quickly through a transition state with two $8 \mathrm{c}$ site vacancies $\left(\mathrm{V}_{\mathrm{o}}\right)$, two $<110>$ interstitials $\left(\mathrm{O}^{\prime}\right)$, and two $<111>$ interstitials (O”), which resembles the $2: 2: 2$ Willis defect cluster. Between the transitions, the split di-interstitials cluster remain relatively stable. At some point during the MD run, the split di-interstitial cluster was temporarily transformed into a di-interstitial (i.e., two mono-interstitials at neighboring locations), but transformed back to a split di-interstitial cluster, which does not involve the $2: 2: 2$ Willis configuration. Figure 3 shows the MD profiles of the potential energy, temperature, and pressure with time as the cluster migrates. Split diinterstitial clusters constitute the majority of the simulation time, and the Willis cluster is only a transition state occurring in a small percentage of the time. In order to check how individual split diinterstitials clusters interact with each other, similar moleculardynamics simulations of $\mathrm{UO}_{2.13}$ and $\mathrm{UO}_{2.19}$ were completed at $2000 \mathrm{~K}$ with two and three di-interstitials in a $2 \times 2 \times 2$ supercell, respectively. At the beginning of the simulations, each individual cluster behaved similarly to a single split di-interstitial cluster. However, once they were in contact and aggregated, the mobility of the clusters slowed because of complex interactions between them. During the migration of the interacting clusters, one of the split diinterstitial clusters had to be activated to a transition state similar to the Willis cluster. One important result of the simulations of single and multiple di-interstitial clusters is that the $2: 2: 2$ Willis defect configuration is actually a transition state for migrating clusters at all of the $\mathrm{x}$ values investigated.

While the oxygen defect clusters migrate, the uranium atoms are immobile. With the excess oxygen atoms in the structure, some 

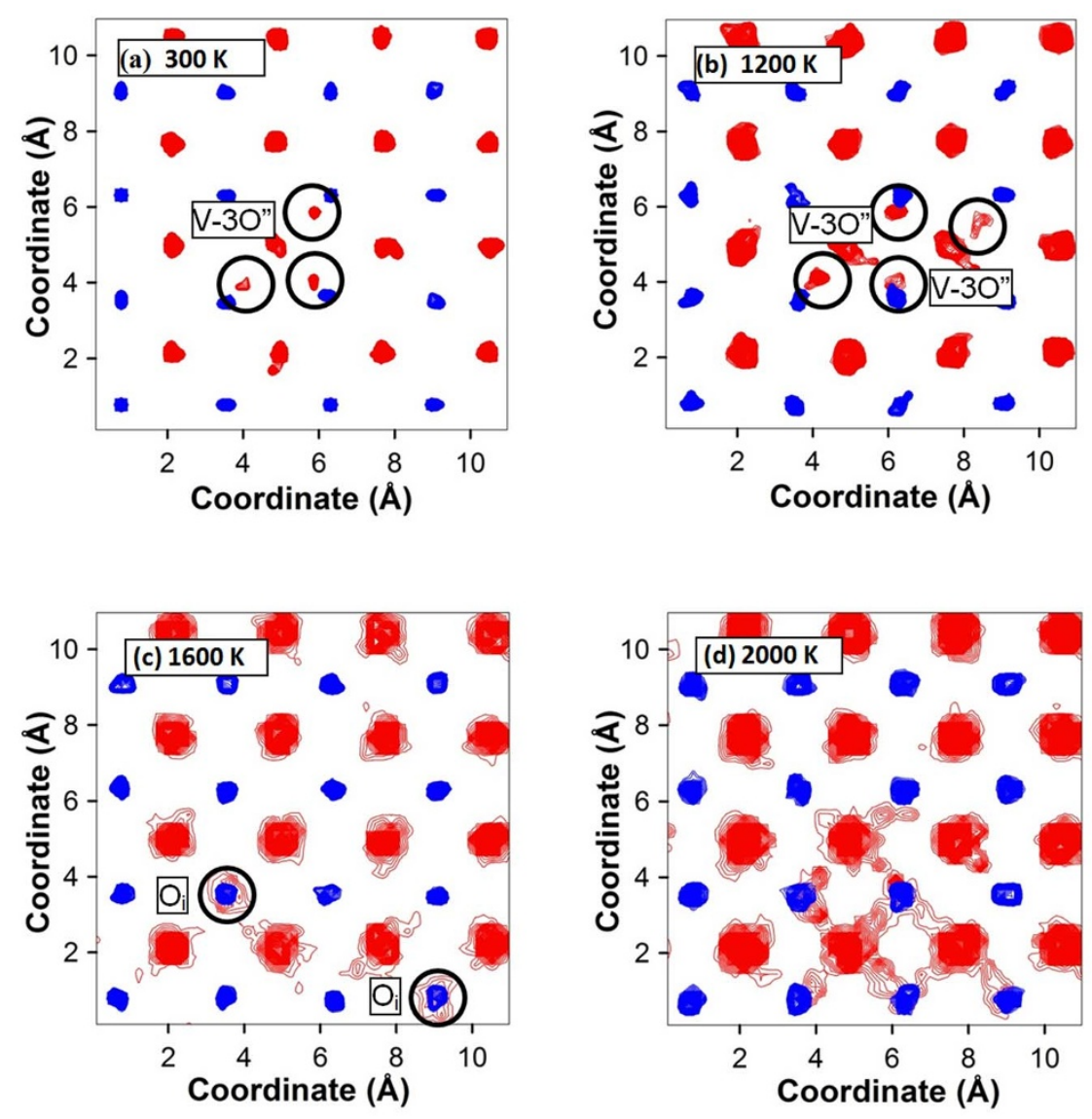

Figure $2 \mid$ Atomic density probability contour maps of $\mathrm{UO}_{2.06}\left(\mathrm{U}_{32} \mathrm{O}_{66}\right)$ projected onto (100) plane, calculated from the $\mathrm{MD}$ trajectories at different temperatures (a: $300 \mathrm{~K}$, b: $1200 \mathrm{~K}, \mathrm{c}: 1600 \mathrm{~K}$, d: $2000 \mathrm{~K}$ ). Circles indicate the locations of the interstitial $\mathrm{O}$ atoms.

uranium atoms are oxidized from $\mathrm{U}^{4+}$ to $\mathrm{U}^{5+}$ by transferring an electron from uranium to oxygen. An interesting observation is that, at low temperatures $(300 \mathrm{~K}-800 \mathrm{~K})$, the $\mathrm{U}^{5+}$ atoms are mostly adjacent to the defect cluster, while at temperatures above $1200 \mathrm{~K}$, the $5+$ oxidation state occurs at all uranium lattice sites over the course of the simulation time of the 3 ps as shown in Figure S1. The fact that the location of $\mathrm{U}^{5+}$ is not constrained by the interstitial $\mathrm{O}$ atoms at temperatures lower than the temperatures at which the di-interstitial cluster becomes mobile suggests that the charge mobility is decoupled from the interstitial oxygen mobility. It seems that the oxidation state of $\mathrm{U}$ atoms changes between $\mathrm{U}^{4+}$ and $\mathrm{U}^{5+}$ at a faster rate than oxygen cluster migration through the $U$ lattice.

In order to reconcile these computational results with the early neutron diffraction data, average occupancy numbers and displacements of O' and O" interstitials were calculated over the trajectory for each composition and are listed in Table 1. For $\mathrm{UO}_{2.06}$, at low temperatures, the interstitials are mainly displaced along $<111>$. As the temperature increases, the O'/O" ratio increases. As expected, the displacements increase with temperature as well. As $\mathrm{x}$ increases from $2.06,2.13$, to 2.19 at $2000 \mathrm{~K}$, the O'/O" ratio increases because the interstitial $\mathrm{O}$ atoms joining neighboring di-interstitial clusters are mainly displaced along $\langle 110\rangle$. The calculated ratios of O' and O" occupancies for $\mathrm{UO}_{2.13}, 0.15: 0.09$, is consistent with the experimental values of $0.08-0.33: 0.10-0.16$ for $\mathrm{UO}_{2.11}-\mathrm{UO}_{2.13}$ based on neutron diffraction ${ }^{3-5}$. The calculated displacements for $\mathrm{UO}_{2.13}$ are $0.77 \pm 0.21 \AA$ and $0.92 \pm 0.20 \AA$ along $<110\rangle$ and $<111>$, respectively, as compared with experimental values of $0.85 \pm 0.08 \AA$ and $1.04 \pm 0.10 \AA$ for $\mathrm{UO}_{2.12}$ at $1073 \mathrm{~K}^{4}$. Note that the calculations were done at a higher temperature for the composition, which is necessary to have adequate statistical averages in a short MD simulation. However, the temperature only has a small effect on the values of

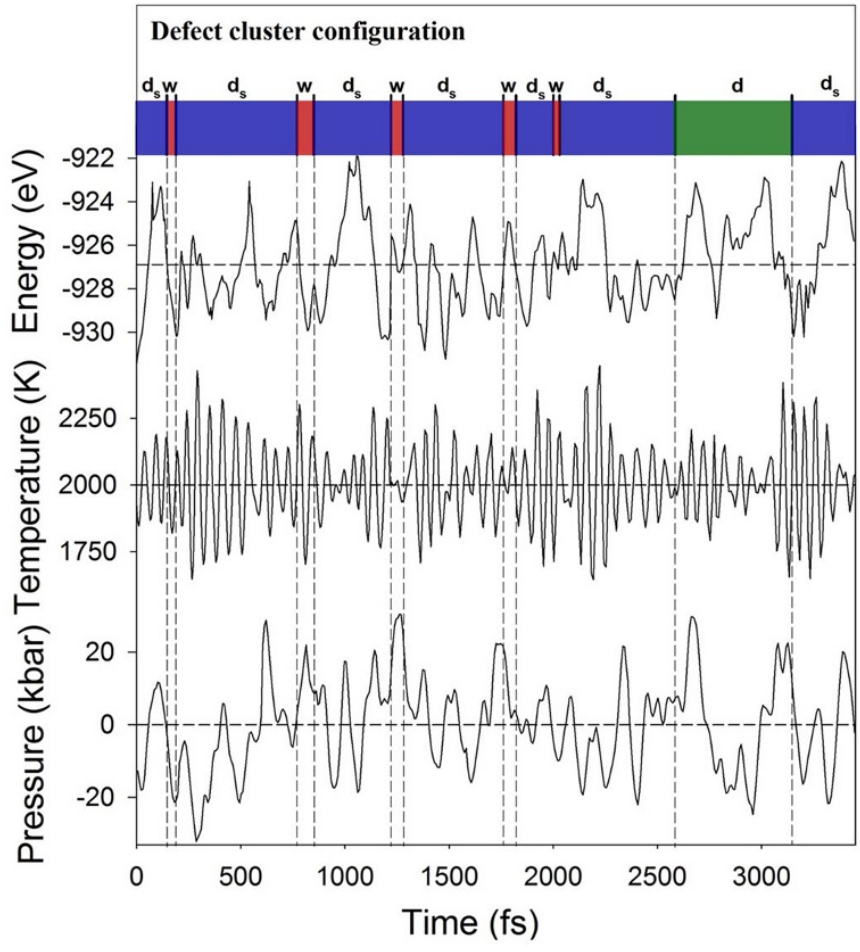

Figure 3 Potential energy, temperature, and pressure profiles over the course of a $\sim 3.5$ ps MD simulation of $\mathrm{UO}_{2.06}\left(\mathrm{U}_{32} \mathrm{O}_{66}\right)$ at $2000 \mathrm{~K}$. Top panel shows defect cluster configurations of different types: $\mathrm{d}_{\mathrm{s}}$ : split diinterstitial (blue), w: Willis $2: 2: 2$ defect cluster (red), d: di-interstitial (green). 
Table $1 \mid$ Average occupancy numbers and displacements of $<110>$ and $<111>$ interstitial $O$ atoms in $\mathrm{UO}_{2+x}$ with different $x$ values at different temperatures. $v$ is the displacement in $\langle 110\rangle$ directions and $w$ is in the $\langle 111\rangle$ directions

\begin{tabular}{lccccc} 
Composition & \multicolumn{3}{c}{$\mathrm{UO}_{2.06}\left(\mathrm{U}_{32} \mathrm{O}_{66}\right)$} & $\mathrm{UO}_{2.13}$ & $\mathrm{UO}_{2.19}$ \\
\hline Temperature & $300 \mathrm{~K}$ & $1200 \mathrm{~K}$ & $2000 \mathrm{~K}$ & $2000 \mathrm{~K}$ & $2000 \mathrm{~K}$ \\
\hline $\mathrm{O} / \mathrm{O}^{\prime} / \mathrm{O}^{\prime \prime}$ & $1.97 / 0.00 / 0.09$ & $1.95 / 0.05 / 0.06$ & $1.95 / 0.06 / 0.05$ & $1.89 / 0.15 / 0.09$ & $1.90 / 0.19 / 0.10$ \\
$v<110>$ & $0.67 \pm 0.07$ & $0.71 \pm 0.18$ & $0.74 \pm 0.27$ & $0.77 \pm 0.21$ & $0.72 \pm 0.24$ \\
$w<111>(\dot{A})$ & $0.78 \pm 0.09$ & $0.87 \pm 0.19$ & $0.90 \pm 0.25$ & $0.92 \pm 0.20$ & $0.90 \pm 0.24$ \\
\hline
\end{tabular}

both the $\mathrm{O}^{\prime} / \mathrm{O}^{\prime \prime}$ ratio and displacements as the temperature increases from $1200 \mathrm{~K}$ to $2000 \mathrm{~K}$ as shown in Table 1 for $\mathrm{UO}_{2.06}$. The lower temperature is comparable to the experimental temperature conducted at $1073 \mathrm{~K}^{4}$. The probability distributions of the angle between $<111>$ and the displacement direction of interstitial oxygen atom are shown in Figure S2. The calculated result suggests that the $2: 2: 2$ Willis cluster model for $\mathrm{UO}_{2.11-13}$ does account for the numerical fraction of the O' and O" interstitials. However, the Willis model does not represent the local defect configuration of defect clusters. The often assumed Willis $2: 2: 2$ defect configuration is, in fact, a transition state for the migration of a split di-interstitial cluster in the hyperstoichiometric $\mathrm{UO}_{2}$. A careful review of all the trajectories shows that the average structure of $\mathrm{UO}_{2+\mathrm{x}}$ involves a combination of defect structures including the split di-interstitial, di-interstitial, mono-interstitial, and Willis cluster, and the latter serves as a transition state for a fast diffusion of the defect cluster (Figure 4). Spectroscopic techniques such as vibrational spectroscopy can be used to validate local structure configuration of different types of defect clusters, and property measurements such as electron and ionic conductivity can be used to test if the charge transport between

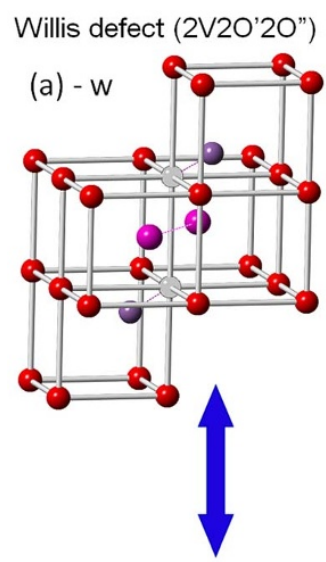

Split di-interstitial (V3O")

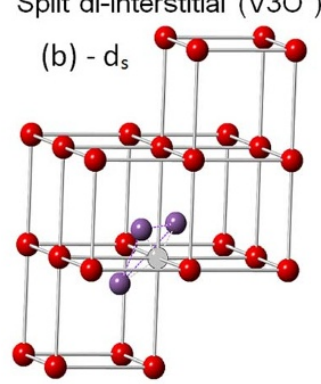

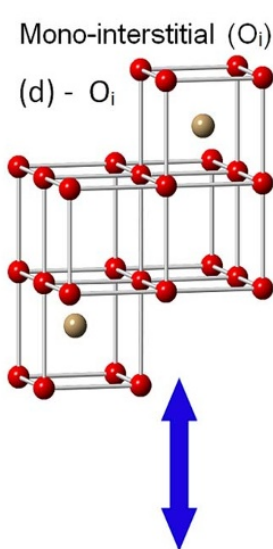

Di-interstitials $\left(2 \mathrm{O}_{\mathrm{i}}\right)$

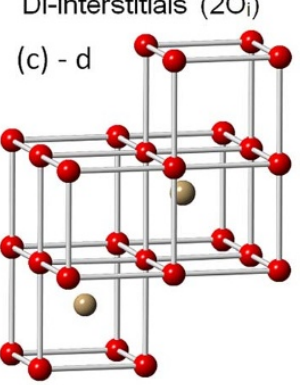

Figure $4 \mid$ Defect models for oxygen interstitials in $\mathrm{UO}_{2+\mathrm{x}}$. The Willis defect cluster (a) serves as a transition state for rapid diffusion of the split di-interstitial defect (b). The latter can also migrate through a diinterstitial (c). Di-interstitial can dissociate to two immobile monointerstitials (d).
$\mathrm{U}^{4+}$ and $\mathrm{U}^{5+}$ is activated at a lower temperature than the oxygen migration.

\section{Methods}

The first-principles calculations were based on the Density Functional Theory and plane wave basis sets as implemented in VASP ${ }^{27}$. The Projector-Augmented Wave method and exchange-correlation as parameterized by the Perdew-Wang 91 functional were applied in the Generalized Gradient Approximation ${ }^{28,29}$. For U, 14 electrons are treated as valence electrons and the core electrons have [Xe, 5d, 4f] configuration. For O, 6 electrons are treated as valence electrons and the core has [He]. On-site Coulomb interaction $(\mathrm{U}=4.5, \mathrm{~J}=0.51)$ with the rotational invariant Liechtenstein approach ${ }^{30}$, fully relativistic calculation for the core-electrons, and the scalar relativistic approximation for the valence electrons were employed to account for electron correlation and relativistic effects ${ }^{31}$. The $U$ and $J$ values are based on the experiment ${ }^{32}$, and these values have been used in a number of most recent publications of DFT $+\mathrm{U}$ studies of $\mathrm{UO}_{2}{ }^{6,33,34}$. However, other different $\mathrm{U}$ and $\mathrm{J}$ values have also been used in the literature ${ }^{35,36}$, depending on how $\mathrm{U}$ and $\mathrm{J}$ values were determined. We have used $\mathrm{U}=3.8 \mathrm{eV}$ and $\mathrm{J}=0.4 \mathrm{eV}$ in previous publications $\mathrm{s}^{36,37}$, the results are comparable with those using $\mathrm{U}=4.5 \mathrm{eV}$ and $\mathrm{J}=0.51 \mathrm{eV}$. In this study, for the reason of comparison and consistency with the majority of the literature, the values based on the experiment were used. The details and performance of the methods on the calculations of crystal structure and electronic structure of $\mathrm{UO}_{2+\mathrm{x}}$ are summarized in a previous publication ${ }^{36}$. All the calculations were performed with a supercell of $2 \times 2 \times 2$ unit cells of $\mathrm{UO}_{2}$ and the energy cut-off for the plane-wave basis was set to $520.00 \mathrm{eV} .3 \times 3 \times 3 \mathrm{k}$-point grids were used for the static calculations and $\Gamma$ point for the molecular dynamics simulations. Ferromagnetic ordering without spinorbit coupling was used in the molecular-dynamics simulations. Antiferromagnetic and ferromagnetic configurations were both used in the static calculations to test the effect of the spin configurations on the calculations. The optimized volumes were used for static calculations. For dynamics simulations, both optimized volumes and relaxed volumes at 1 bar and high temperatures were used. Molecular-dynamics simulations were performed using a NVT ensemble with the Nosé-thermostat for 2-3 ps for equilibration, which was checked by monitoring a number of parameters including temperature, pressure, potential energy of the systems, and kinetic energy of both $\mathrm{U}$ and $\mathrm{O}$ sublattices. The time step is $0.5 \mathrm{fs}$ for low-temperature simulations $(300-800 \mathrm{~K})$ and $0.25 \mathrm{fs}$ for high-temperature (1200-2000 K). It is necessary to carry out the simulations at high temperatures, at which multiple migration events can be observed and statistical meaningful averages can be obtained in short molecular dynamics simulations. The total energy drift was $\sim(2-8) \mathrm{meV} /$ atom/ps at low temperatures and increased to $\sim 25(15-50) \mathrm{meV} / \mathrm{atom} / \mathrm{ps}$ at high temperatures. For $\mathrm{UO}_{2+\mathrm{x}}$ with unpaired $5 \mathrm{f}$ electrons, it was extremely challenging to achieve both high accuracy and adequate sampling with enough statistics of the systems. Extensive tests were performed to balance between the accuracy and duration of the simulations by systematically tuning simulation parameters including those related to convergences, real space projection, and time step. After equilibrations, simulations run up to $4 \mathrm{ps}$ for statistical analysis.

1. Olander, D. R. Mechanistic interpretations of $\mathrm{UO}_{2}$ oxidation. J. Nucl. Mater. 252, 121-130 (1998)

2. Thompson, W. T. et al. Thermodynamic treatment of uranium dioxide based nuclear fuel. Int. J. Mater. Res. 98, 1004-1011 (2007).

3. Willis, B. T. M. Positions of Oxygen Atoms in $\mathrm{UO}_{2.13}$. Nature 197, 755-\& (1963).

4. Willis, B. T. M. Defect Structure of Hyper-Stoichiometric Uranium-Dioxide. Acta Crystallogr., Sect. A: Found. Crystallogr. 34, 88-90 (1978).

5. Murray, A. D. \& Willis, B. T. M. A neutron-diffraction study of anion clusters in onstoichiometric uranium-dioxide. J. Solid State Chem. 84, 52-57 (1990).

6. Geng, H. Y. et al. Point defects and clustering in uranium dioxide by LSDA $+\mathrm{U}$ calculations. Phys. Rev. B 77, 104120 (2008).

7. Andersson, D. A. et al. Cooperativity among defect sites in $\mathrm{AO}_{2+\mathrm{x}}$ and $\mathrm{A}_{4} \mathrm{O}_{9}$ ( $A=\mathrm{U}, \mathrm{Np}, \mathrm{Pu}$ ): Density functional calculations. Phys. Rev. B 79 (2009).

8. Dorado, B. et al. Stability of oxygen point defects in $\mathrm{UO}_{2}$ by first-principles DFT plus $\mathrm{U}$ calculations: Occupation matrix control and Jahn-Teller distortion. Phys. Rev. B 82, 035114 (2010).

9. He, H. \& Shoesmith, D. Raman spectroscopic studies of defect structures and phase transition in hyper-stoichiometric $\mathrm{UO}_{2+\mathrm{x}}$. Phys. Chem. Chem. Phys. 12, 8108-8117 (2010). 
10. Crocombette, J.-P., Torumba, D. \& Chartier, A. Charge states of point defects in uranium oxide calculated with a local hybrid functional for correlated electrons. Phys. Rev. B 83, 184107 (2011).

11. Bai, X. M. et al. Migration mechanisms of oxygen interstitial clusters in $\mathrm{UO}_{2}$ J. Phys.: Condens. Matter 25, 015003 (2013).

12. Fink, J. K. Thermophysical properties of uranium dioxide. J. Nucl. Mater. 279, $1-18$ (2000).

13. Stan, M. Multi-Scale Models and Simulations of Nuclear Fuels. Nucl. Eng. and Technol. 41, 39-52 (2009).

14. White, J. T. \& Nelson, A. T. Thermal conductivity of $\mathrm{UO}_{2+\mathrm{x}}$ and $\mathrm{U}_{4} \mathrm{O}_{9-\mathrm{y}}$. J. Nucl. Mater. 443, 342-350 (2013).

15. Grimes, R. W. \& Catlow, C. R. A. The stability of fission-products in uraniumdioxide. Philos. Trans. R. Soc. London, A 335, 609-634 (1991).

16. Lucuta, P. G. et al. Microstructural features of simfuel - simulated high-burnup $\mathrm{UO}_{2}$-based nuclear-fuel. J. Nucl. Mater. 178, 48-60 (1991).

17. Nogita, K. \& Une, K. Thermal recovery of radiation defects and microstructural change in irradiated $\mathrm{UO}_{2}$ fuels. J. Nucl. Sci. Technol. 30, 900-910 (1993).

18. Shoesmith, D. W. Fuel corrosion processes under waste disposal conditions. J. Nucl. Mater. 282, 1-31 (2000).

19. He, H. M. et al. Determination of local corrosion kinetics on hyper-stoichiometric $\mathrm{UO}_{2+\mathrm{x}}$ by scanning electrochemical microscopy. J. Electrochem. Soc. 156 C87-C94 (2009).

20. Catlow, C. R. A. Point-defect and electronic properties of uranium-dioxide. Philos. Trans. R. Soc. London, A 353, 533-561 (1977).

21. Crocombette, J. P. et al. Plane-wave pseudopotential study of point defects in uranium dioxide. Phys. Rev. B 64, 104107 (2001).

22. Geng, H. Y. et al. Ab initio investigation on oxygen defect clusters in $\mathrm{UO}_{2+\mathrm{x}} \cdot$ Appl. Phys. Lett. 93 (2008)

23. Andersson, D. A. et al. Role of di-interstitial clusters in oxygen transport in $\mathrm{UO}_{2+\mathrm{x}}$ from first principles. Phys. Rev. B 80 (2009).

24. Govers, K. et al. Comparison of interatomic potentials for $\mathrm{UO}_{2}$. Part I: Static calculations. J. Nucl. Mater. 366, 161-177 (2007).

25. Ichinomiya, T. et al. Temperature accelerated dynamics study of migration process of oxygen defects in $\mathrm{UO}_{2}$. J. Nucl. Mater. 384, 315-321 (2009).

26. Henkelman, G., Uberuaga, B. P. \& Jonsson, H. A climbing image nudged elastic band method for finding saddle points and minimum energy paths. J. Chem. Phys. 113, 9901-9904 (2000).

27. Kresse, G. \& Furthmuller, J. Vienna ab-initio simulation package (Universitat Wein, 2004).

28. Blöchl, P. E. Projector augmented-wave method. Phys. Rev. B 50, 17953-17979 (1994).

29. Perdew, J. P. et al. Atoms, molecules, solids, and surfaces: Applications of the generalized gradient approximation for exchange and correlation. Phys. Rev. B 46, 6671-6687 (1992)
30. Liechtenstein, A. I., Anisimov, V. I. \& Zaanen, J. Density-Functional Theory and Strong-Interactions - Orbital Ordering in Mott-Hubbard Insulators. Phys. Rev. B 52, R5467-R5470 (1995).

31. Hafner, J. Ab-initio simulations of materials using VASP: Density-functional theory and beyond. J. Comput. Chem. 29, 2044-2078 (2008).

32. Dudarev, S. L., Manh, D. N. \& Sutton, A. P. Effect of Mott-Hubbard correlations on the electronic structure and structural stability of uranium dioxide. Philosophical Magazine B-Physics of Condensed Matter Statistical Mechanics Electronic Optical and Magnetic Properties 75, 613-628 (1997).

33. Meredig, B. et al. Method for locating low-energy solutions within DFT plus U. Phys. Rev. B 82, 195128 (2010).

34. Andersson, D. A. et al. $\mathrm{U}$ and $\mathrm{Xe}$ transport in $\mathrm{UO}_{2 \pm \mathrm{x}}$ : Density functional theory calculations. Phys. Rev. B 84, 054105 (2011).

35. Yu, J. G., Devanathan, R. \& Weber, W. J. First-principles study of defects and phase transition in $\mathrm{UO}_{2}$. Journal of Physics-Condensed Matter 21, 435401 (2009).

36. Wang, J., Ewing, R. C. \& Becker, U. Electronic structure and stability of hyperstoichiometric $\mathrm{UO}_{2+\mathrm{x}}$ under pressure. Phys. Rev. B 88, 024109 (2013).

37. Wang, J. \& Becker, U. Activation energetics of actinide diffusion in $\mathrm{UO}_{2}$ from first-principles calculations. J. Nucl. Mater. 433, 424-430 (2013).

\section{Acknowledgments}

This work was supported by the Center for the Materials Science of Actinides, an Energy Frontier Research Center, funded by the U.S. Department of Energy (DE-SC0001089). The computational work was supported by NERSC (DE-AC02-05CH11231) and XSEDE (TG-DMR080047N and TG-DMR100034).

\section{Author contributions}

J.W., R.C.E., and U.B. participated in conceiving and designing the computations. J.W carried out the computations. J.W., R.C.E., and U.B. have reviewed, discussed and approved the results and conclusions of this report.

\section{Additional information}

Supplementary information accompanies this paper at http://www.nature.com/ scientificreports

Competing financial interests: The authors declare no competing financial interests.

How to cite this article: Wang, J.W., Ewing, R.C. \& Becker, U. Average structure and local configuration of excess oxygen in $\mathrm{UO}_{2+\mathrm{x}}$. Sci. Rep. 4, 4216; DOI:10.1038/srep04216 (2014).

This work is licensed under a Creative Commons AttributionNonCommercial-ShareAlike 3.0 Unported license. To view a copy of this license, visit http://creativecommons.org/licenses/by-nc-sa/3.0 